\title{
CDKN2A NM_000077.4:C.389T>A
}

National Cancer Institute

\section{Source}

National Cancer Institute. CDKN2A NM 000077.4:C.389T>A. NCI Thesaurus. Code C146945.

A nucleotide substitution at position 389 of the coding sequence of the CDKN2A gene where thymine has been mutated to adenine. 